\section{Robust Control of Robots via Linear Estimated State Feedback}

\author{
Harry Berghuis and Henk Nijmeijer
}

\begin{abstract}
In this note we propose a robust tracking controller for robots that requires only position measurements. The controller consists of two parts: a linear observer part that generates an estimated error state from the error on the joint position and a linear feedback part that utilizes this estimated state. It is shown that this computationally efficient controller yields semi-global uniform ultimate boundedness of the tracking error. An interesting feature of the controller is that it straightforwardly extends recent results on robust control of robots by linear state feedback to linear estimated-state feedback.
\end{abstract}

\section{INTRODUCTION}

Over the last decade, a lot of research effort has been put into the design of sophisticated control strategies for robots, see, for instance, [12]. In spite of these efforts, virtually all industrial robot systems today are still controlled by some kind of linear state feedback [1], [14]. The reasons for this are threefold. First, the linear state feedback (in literature frequently referred to as PD controller) is computationally simple and does not require any model knowledge, which makes it attractive from the viewpoint of implementation. Second, practice has proved that the PD controller is robust to disturbances like friction and load torques, which represents a prerequisite for the realm of applications. Third, since industrial robots are typically overdesigned in the sense that heavy and consequently rigid links are used together with high-gear transmission mechanisms [1], they can be described by linear and decoupled dynamics, for which linear state-feedback generally provides acceptable performance.

Owing to the increasing demands on productivity and efficiency of robots, there has been, in recent years, a tendency to develop fast lightweight robot constructions actuated by direct-drive motors. Contrary to the traditional manipulator, these newly developed robot systems are characterized by highly nonlinear and coupled dynamics. As was shown in [8], [10], [15], and more recently in [13], even for such systems the classical PD controller may work reasonably well. In particular, in [13] it is shown that in the presence of arbitrary but bounded nonlinearities in the system dynamics, the PD controller yields uniform ultimate boundedness (also known as practical stability) of the tracking errors; that is the error state tends in finite time to a bounded region around zero. An interesting feature of this stability result is that the authors can provide a relationship between the bound on the tracking errors and the PD feedback gains, for any initial condition. Moreover, it is demonstrated that the ultimate error bound can be made arbitrarily small by increasing the controller gains.

One characteristic feature of the state-feedback controller is that it requires both position and velocity measurements. In practice,

Manuscript received August 24, 1993; revised December 10, 1993. This work was supported in part by the Netherlands' Technology Foundation (STW).

H. Berghuis was with the Department of Electrical Engineering, University of Twente, Netherlands and is now with Hollandse Signaalapparaten B.V., Afdeling DNCS-RS, PO Box 42, 7550 GD Hengelo, Netherlands.

$\mathrm{H}$. Nijmeijer is with the Department of Applied Mathematics, University of Twente, PO Box 217, 7500 AE Enschede, Netherlands.

IEEE Log Number 9403958. however, this requirement is generally not fulfilled; although in robotic applications today high-precision sensors are used to obtain position information, velocity sensoring equipment is frequently omitted due to the savings in cost, volume, and weight that can be obtained [9]. For these reasons, a number of model-based robot control methods have been proposed recently that evade the velocity measurement problem by integrating a velocity observer in the control loop (e.g., [3], [4], [11]). These methods require exact knowledge of the nonlinear robot dynamics, which, in practice is generally not available. Motivated by this, Canudas de Wit and Fixot [5] have addressed the robust tracking control problem of robots using only position feedback. These authors combine a nonlinear switching type control method with a sliding mode velocity observer to face bounded uncertainties in the robot dynamics.

In this paper we present a novel approach to the robust control problem stated above. This approach originates from a strategy for combined controller-observer design for robots that we recently proposed in [3]; see also [2]. The rationale underlying this strategy is to extend in a natural way the passivity methodology to statefeedback robot control (cf. [12] and references therein) to the case that only joint position measurements are present. This allows us to develop a controller that consists of two parts:

1) a linear observer that generates an estimated error state from the position error

2) a linear feedback controller that employs the estimate of the error state.

By using stability analysis techniques that are similar to the ones in [13], it is proved that this linear estimated-state feedback controller provides uniform ultimate boundedness of the closed-loop error dynamics. As in [13], we given an explicit relation between the bound on the error state and the controller and observer feedback gains. This, together with the fact that the linear estimated-state feedback controller is easily implementable and needs only position information, makes the proposed controller particularly interesting from a practical point of view.

This paper is organized as follows. In Section II some mathematical preliminaries are given that support the stability analysis in the following sections. The proposed robot controller is introduced in Section III, together with its stability properties. Section IV contains a discussion of some characteristics of the novel control approach, and finally we give conclusions. Standard notation is used. In particular, vector norms are Euclidean, and for matrices the induced norm $\|A\|=\sqrt{\lambda_{\max }\left(A^{T} A\right)}$ is employed, with $\lambda_{\max }(\cdot)$ the maximum eigenvalue. Moreover, for any positive definite matrix $A(x)$ and for all $x$ we denote by $A_{m}$ and $A_{M}$ the minimum and maximum eigenvalue of $A(x)$, respectively.

\section{Mathematical Preliminaries}

This section presents a stability result that plays a central role in the sequel. This result is a modified version of a theorem by Chen and Leitmann [6] (see also [13]), which basically states that a system is uniformly ultimately bounded if it has a Lyapunov function whose time-derivative is negative definite in an annulus of a certain width around the origin. For the sake of brevity, the proof is omitted. The following lemma is useful for the stability analysis.

Lemma 1: Consider the function $g(\cdot): \mathbb{R} \rightarrow \mathbb{R}$

$$
g(y)=\alpha_{0}+\alpha_{1} y-\alpha_{2} y^{2}, \quad y \in \mathbb{R}^{+}
$$


where $\alpha_{i}>0 . i=0,1,2$. Then $g(y)<0$ if $y>\eta>0$, where

$$
\eta=\frac{\alpha_{1}+\sqrt{\alpha_{1}^{2}+4 \alpha_{0} \alpha_{2}}}{2 \alpha_{2}}
$$

Proposition 1: Let $x(t) \in \mathbb{R}^{m}$ be the solution of the differential equation

$$
\dot{x}(t)=f(x(t), t) \quad x\left(t_{0}\right)=x_{0}
$$

and assume there exists a function $V(x(t), t)$ that satisfies

$$
\begin{gathered}
P_{m}\|x(t)\|^{2} \leq V(x(t), t) \leq P_{M}\|x(t)\|^{2} \\
\dot{V}(x(t), t) \leq g(\|x(t)\|)<0 \text { for all }\|x(t)\|>\eta>0
\end{gathered}
$$

with $P_{m}$ and $P_{M}$ positive constants, $g(\cdot)$ as in (1), and $\eta$ as in (2). Define $\delta \equiv \sqrt{P_{m}^{-1} P_{M}}$ and $d>\delta \eta$. Then $x(t)$ is uniformly ultimately bounded, that is

$$
\left\|x_{0}\right\| \leq r \rightarrow\|x(t)\| \leq d \quad \text { for all } t \geq t_{0}+T(d, r)
$$

where

$$
T(d, r)= \begin{cases}0 & r \leq R \\ \frac{P_{M} r^{2}-P_{m} R^{2}}{\alpha_{2} R^{2}-\alpha_{1} R-\alpha_{0}} & r>R\end{cases}
$$

and $R=\delta^{-1} d$

\section{Linear Estimated State-FeEdBack Controller}

The general equations describing the dynamics of an $n$ degreesof-freedom rigid robot manipulator are given by [14]

$$
M(q) \ddot{q}+C(q, \dot{q}) \dot{q}+G(q)+F(\dot{q})+T=\tau
$$

where $q$ is the $[n \times 1]$ vector of generalized coordinates, $M(q)=$ $M(q)^{T}>0$ the $[n \times n]$ positive definite inertia matrix, $C(q, \dot{q}) \dot{q}$ the Coriolis and centrifugal torques $[n \times 1], G(q)$ the gravitational torques $[n \times 1], F(\dot{q})$ the friction torques $[n \times 1], T$ an $[n \times 1]$ vector of load disturbances, and $\tau$ the $[n \times 1]$ vector of control torques. The matrix $C(q, \dot{q})$ is defined via the Christoffel symbols [12], which implies that $\dot{M}(q)-2 C(q, \dot{q})$ is skew symmetric. We use the following property.

Property 1: For revolute robots, $M(q) . C(q, \dot{q})$, and $G(q)$ are unbounded w.r.t. $q$, i.e., (cf. [7])

$$
\begin{gathered}
0<M_{m} \leq\|M(q)\| \leq M_{M} \text { for all } q \\
\|C(q, x)\| \leq C_{M}\|x\| \quad \text { for all } q, x \\
\|G(q)\| \leq G_{M} \text { for all } q .
\end{gathered}
$$

In addition, the friction and load disturbance torques are bounded by (cf. [7])

$$
\|F(\dot{q})\| \leq F_{1, M}+F_{2, M}\|\dot{q}\| \text { for all } \dot{q}
$$

$$
\|T\| \leq T_{M} .
$$

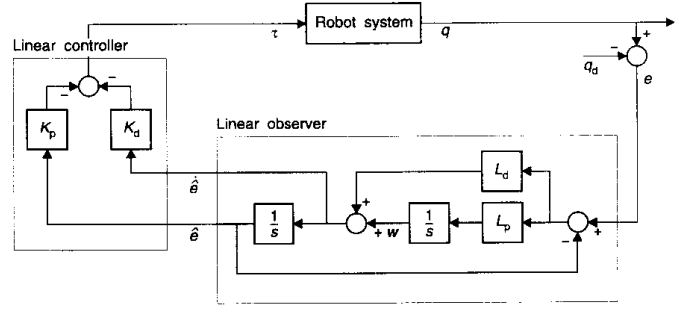

Fig. 1. Proposed linear controller-observer combination.

To solve the tracking control problem for (6) using position feedback only, consider the linear output-feedback robot control system (see Fig. 1)

$$
\begin{gathered}
\text { Controller }\left\{\tau=-K_{d} \dot{\hat{e}}-K_{p} \hat{e}\right. \\
\text { Observer }\left\{\begin{array}{l}
\dot{\hat{e}}=w+L_{d}(e-\hat{e}) \\
\dot{w}=L_{p}(e-\hat{e})
\end{array}\right.
\end{gathered}
$$

where $q_{d}(t)$ represents the desired path to be tracked by the robot system, $\hat{e} \equiv \hat{q}-q_{d}, K_{p}=K_{p}^{T}>0$ the controller proportional gain, $K_{d}=K_{d}^{T}>\phi$ the controller derivative gain, $L_{p}=L_{p}^{T}>0$ the observer proportional gain, and $L_{d}=L_{d}^{T}>0$ the observer derivative gain. This control system consists of two parts: a linear observer part $(8 \mathrm{~b})$ that generates an estimated error state $\left[\dot{\hat{e}}^{T} \cdot \hat{e}^{T}\right]^{T}$ from the tracking error $e$ and a linear controller part (8a) that utilizes this estimated error state in the feedback loop.

Let us make the following assumption on the structure of $K_{p}^{-}, K_{d}$. $L_{p}$, and $L_{d}$.

Assumption $1: K_{p}, K_{d}$, and $L_{p}, L_{d}$ satisfy respectively

$$
\begin{gathered}
K_{p}=\lambda K_{d}, \quad K_{d}=\left(k_{d}+\gamma\right) I \\
L_{p}=\lambda l_{d}, \quad L_{d}=\left(l_{d}+\lambda\right) I
\end{gathered}
$$

where $\lambda>0, k_{d}>0, \gamma>0$ and $l_{d}>0$ all scalar.

In addition, we require the following assumptions.

Assumption 2: The desired trajectory signals $\dot{q}_{d}(t)$ and $\ddot{q}_{d}(t)$ are bounded by $V_{M}$ and $A_{M}$ respectively, i.e.,

$$
V_{M}=\sup _{t}\left\|\dot{q}_{d}(t)\right\|, \quad A_{M}=\sup _{t}\left\|\ddot{q}_{d}(t)\right\| .
$$

Then our main result can be formulated as the following theorem.

Theorem 1: Consider the linear output-feedback robot controller (8) in closed loop with (6). Define $x(t)^{T}=\left[\dot{e}(t)^{T}(\lambda e(t))^{T} \dot{\tilde{q}}(t)^{T}\right.$ $\left.(\lambda \tilde{q}(t))^{T}\right]$, where $e \equiv q-q_{d}, \tilde{q} \equiv q-\hat{q}$, and assume that $\left\|x_{0}\right\|$ represents an upper bound on the initial error state $x(0)$. Under the conditions

$$
\begin{gathered}
k_{d}>\lambda M_{M} \\
l_{d}>2 M_{m}^{-1}\left\{k_{d}+\gamma\right\} \\
\gamma>2 \epsilon^{-1}\left(\beta_{0}+\beta_{1}(\delta \mu)+\beta_{2}(\delta \mu)^{2}\right)
\end{gathered}
$$

where

$$
\beta_{0}=M_{M} A_{M}+C_{M} V_{M}^{2}+G_{M}+F_{1, M}+F_{2, M} V_{M}+T_{M}
$$

$$
\beta_{1}=2(1+\sqrt{2}) C_{M} V_{M}+F_{2, M}
$$




$$
\begin{gathered}
3_{2}=(1+\sqrt{2}) C_{M} \\
\delta=3 \sqrt{2\left(\lambda . M_{m}\right)^{-1} k_{d}} \\
\mu=\max \left\{\bar{\eta},\left\|x_{0}\right\|\right\}
\end{gathered}
$$

and $\epsilon$ and $\bar{\eta}$ are positive constants that satisfy

$$
\begin{gathered}
\epsilon<\beta_{2}^{-1}\left(k_{d}-\lambda M_{M}\right) \\
\bar{\eta}=\frac{\epsilon 3_{1}+\sqrt{\left(\epsilon \beta_{1}\right)^{2}+4 \epsilon \beta_{0}\left(k_{d}-\lambda M_{M}-\epsilon \beta_{2}\right)}}{2\left(k_{d}-\lambda M_{M}-\epsilon \beta_{2}\right)}
\end{gathered}
$$

the closed-loop system is uniformly ultimately bounded, with

$$
\|x(t)\| \leq \delta \bar{\eta} \quad \text { for all } t \geq T\left(\delta \bar{\eta} .\left\|x_{0}\right\|\right)
$$

where $T(\cdot)$ defined in (5). In the limiting case that $\epsilon \rightarrow 0$, and consequently $\gamma \rightarrow \infty$ and $l_{d} \rightarrow \infty$, the closed-loop system is asymptotically stable.

Proof: The closed-loop error dynamics (6), (8) are given by

$$
\begin{aligned}
& M(q) \ddot{e}+C(q, \dot{q}) s_{1}+K_{d} s_{1} \\
& \quad=K_{d} s_{2}+C(q, \dot{q}) \lambda e-C\left(q, s_{2}\right) \dot{q}_{d}-\Delta Y(\cdot)
\end{aligned}
$$

$M(q) \dot{s}_{2}+C(q, \dot{q}) s_{2}+\left(l_{d} M(q)-I_{d}\right) s_{2}$

$$
=-K_{d} s_{1}+C\left(q \cdot s_{2}-\dot{q}\right) \dot{e}-\Delta Y(\cdot)
$$

where $s_{1}$ and $s_{2}$ are defined as

$$
\begin{aligned}
& s_{1} \equiv \dot{q}-\dot{q}_{r} \equiv \dot{e}+\lambda e \\
& s_{2} \equiv \dot{q}-\dot{q}_{0} \equiv \dot{\tilde{q}}+\lambda \tilde{q}
\end{aligned}
$$

and the perturbation term $\Delta Y(\cdot)$ satisfies

$\Delta Y\left(q, \dot{q}_{0}, \dot{q}_{d}, \ddot{q}_{d}, t\right)$

$$
=M(q) \ddot{q}_{d}+C\left(q, \dot{q}_{0}\right) \dot{q}_{d}+G(q)+F(\dot{q})+T .
$$

From Property 1 and Assumption 2 it follows that

$$
\begin{aligned}
\|\Delta Y(\cdot)\| \leq & M_{M} A_{M}+C_{M} V_{M}^{2}+G_{M}+F_{1, M}+F_{2, M} V_{M}+T_{M} \\
& +\left\{F_{2, M}+(1+\sqrt{2}) C_{M} V_{M}\right\}\|x\| \\
\equiv & \alpha_{0}+\alpha_{1}\|x\| .
\end{aligned}
$$

Take as a candidate Lyapunov function the function

$$
V(x, t)=\frac{1}{2} x^{T} P(x, t) x
$$

where

$$
\begin{aligned}
& P(x, t)= \\
& {\left[\begin{array}{cc}
{\left[\begin{array}{cc}
M(x, t) & M(x, t) \\
M(x, t) & 2 \lambda^{-1} k_{d}
\end{array}\right]} & 0 \\
0 & {\left[\begin{array}{cc}
M(x, t) & M(x, t) \\
M(x, t) & 2 \lambda^{-1} k_{d}+M(x, t)
\end{array}\right]}
\end{array}\right] .}
\end{aligned}
$$

In the Appendix it is shown that condition (11a) implies that

$$
\frac{1}{2} P_{m}\|x\|^{2} \leq V(x, t) \leq \frac{1}{2} P_{M}\|x\|^{2}
$$

with $P_{m}, P_{M}$ defined by

$$
P_{m}=\frac{1}{3} M_{m}, \quad P_{m}=6 \lambda^{-1} k_{d} .
$$

Along the error dynamics (14), the time-derivative of (18) becomes

$$
\begin{aligned}
\dot{V}(x, t)= & -x^{T} Q(x, t) x-s_{2}^{T}\left(l_{d} M(q)-2 k_{d}-2 \gamma\right) s_{2}+ \\
& -\gamma s_{1}^{T} s_{1}+s_{1}^{T}\left\{-\Delta Y(\cdot)-C\left(q, s_{2}\right) \dot{q}_{d}\right\} \\
& +\dot{e}^{T} C(q, \dot{q})(\lambda e)-\gamma s_{2}^{T} s_{2} \\
& +s_{2}^{T}\left\{-\Delta Y(\cdot)+C\left(q, s_{2}-\dot{q}\right) \dot{e}\right\}
\end{aligned}
$$

with

$$
Q(x, t)=\left[\begin{array}{cc}
{\left[\begin{array}{cc}
k_{d}-\lambda M(x, t) & 0 \\
0 & k_{d}
\end{array}\right]} & 0 \\
0 & {\left[\begin{array}{cc}
k_{d} & 0 \\
0 & k_{d}
\end{array}\right]}
\end{array}\right]
$$

where Assumption 1 and the skew-symmetry of $\dot{M}(q)-2 C(q, \dot{q})$ has been used.

Using Property 1, Assumption 2, condition (11b) and (17), an upper bound on (22) is given by

$$
\begin{aligned}
\dot{V}(x, t) \leq & -Q_{m}\|x\|^{2}+ \\
& -\gamma\left\|s_{1}\right\|^{2}+\left\|s_{1}\right\|\left\{\beta_{0}+\beta_{1}\|x\|+\beta_{2}\|x\|^{2}\right\} \\
& -\gamma\left\|s_{2}\right\|^{2}+\left\|s_{2}\right\|\left\{\beta_{0}+\beta_{1}\|x\|+\beta_{2}\|x\|^{2}\right\}
\end{aligned}
$$

where $Q_{m}=k_{d}-\lambda M_{M}>0$, and $\beta_{i}, i=0,1,2$ as defined in (12a)-(12c). Then the proof can be completed along the lines given in $[13]$.

\section{DisCUSSION}

1) The basic improvement of the result in Theorem 1 in comparison with [13] is that the need of velocity feedback can be eliminated by a simple linear observer system, without affecting the stability properties of the closed loop. This is achieved despite the fact that the conditions on the controller gains remain essentially the same. An obvious additional constraint here is that $l_{d}$, the observer derivative gain, is required to be sufficiently large in order to guarantee uniform ultimate boundedness.

2) Like in [13], the uniform ultimate boundedness result is of local nature because condition (11) depends on the initial condition $x(0)$. Nonetheless, it is important to observe that these conditions can be met for arbitrary $x(0)$. In modern terminology this kind of stability is called semiglobal.

3) The following steps need to be taken to arrive at a stable implementation of the control law (8):

- Determine the robot-specific quantities $M_{m}, M_{M}, C_{M}$, $G_{M}, F_{1, M}, F_{2, M}$, and $T_{M}$.

- Specify upper bounds on the desired velocity and acceleration, $V_{M}$, respectively, $A_{M}$.

- Select $\lambda$ and $k_{d}$, taking into consideration (11a), and compute $\epsilon$ and $\bar{\eta}$ from (12f), (12g).

- Fix an upper bound on the initial error state $x_{0}=$ $\left[\dot{e}(0)^{T}(\lambda e(0))^{T} \dot{\tilde{q}}(0)^{T}(\lambda \tilde{q}(0))^{T}\right]^{T}$.

- Determine $\delta$ and $\mu$ in (12d) and (12e) respectively.

- Choose $\gamma$ and $l_{d}$, under the conditions (11b) and (11c) respectively.

These steps can be used as guidelines for the actual implementation of the control system (8). In this respect it should be emphasized that (13) provides a relation between the ultimate upper bound on the error state and the feedback gains. This relation can appropriately be used to guarantee a prespecified ultimate tracking performance. In practice, however, the tracking accuracy is likely to be better because the bound (13) is generally very conservative (cf. [2]). 
4) Even without knowledge of the bounds in (7) the closed-loop system can be made uniformly ultimately bounded, by selecting $k_{d}, l_{d}$, and $\gamma$ large enough. Hence, there is no need to quantify these bounds a priori.

5) The linear control scheme (8) allows quick response in an online implementation, due to its simplicity. Since this control scheme completely ignores the system dynamics, however, the conditions (11)-(12) may require $k_{d}, l_{d}$, and $;$ to be large to obtain an acceptable tracking performance. Such high gain implementations are not always desirable in practical circumstances. For this reason it may be profitable to add model-based compensation terms to the control input. This also allows to obtain stronger stability properties such as asymptotic stability; see for instance [3], [4], [11].

6) In [2] experimental tests on a two degrees of freedom mechanical manipulator were performed that support the theoretical analysis in Section III. Moreover, comparative experiments in [2] (see also [3]) show that the linear observer outperforms the rather ad-hoc numerical position differentiation algorithm which also can be employed to generate a velocity estimate [9]. This improvement is achieved despite the fact that the additional computations for the linear observer are basically negligible.

\section{CONCLUSIONS}

In the present paper we propose a robust motion control scheme for robots that requires only position measurements. The control scheme consists of a linear feedback controller, which utilizes an estimate of the error state obtained from a simple second-order linear observer. The resulting closed-loop system was proved to possess the practically meaningful uniform ultimate boundedness stability property. Also an explicit relation between the bound on the error state and the design parameters of the control system was given, which can be used to guarantee the desired tracking accuracy. Finally, a constructive design procedure was provided that eases the implementation of the controller.

\section{APPENDIX}

Function (18) can be rewritten as

$$
V(y, t)=\frac{1}{2} y^{T} R(y, t) y
$$

where

$$
y^{T}=\left[\begin{array}{llll}
s_{1}^{T} & (\lambda e)^{T} & s_{2}^{T} & (\lambda \bar{q})^{T}
\end{array}\right]
$$

and

$R(y, t)=$

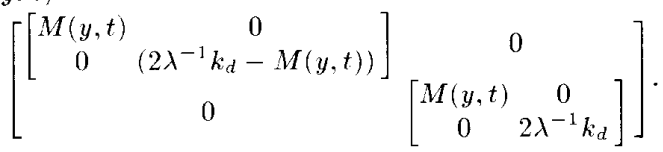

According to (11a)

$$
k_{d}>\lambda M_{M}
$$

Hence

$$
\frac{1}{2} R_{m}\|y\|^{2} \leq V(\cdot) \leq \frac{1}{2} R_{M}\|y\|^{2}
$$

where

$$
R_{m}=M_{m}, \quad R_{M}=2 \lambda^{-1} k_{d} .
$$

By definition

$$
y=T x
$$

with $x^{T}=\left[\dot{e}^{T}(\lambda e)^{T} \dot{\dot{q}}^{T}(\lambda \tilde{q})^{T}\right]$ and

$$
T=\left[\begin{array}{cc}
{\left[\begin{array}{cc}
I & I \\
0 & I
\end{array}\right]} & 0 \\
0 & {\left[\begin{array}{ll}
I & I \\
0 & I
\end{array}\right]}
\end{array}\right]
$$

It can easily be verified that

$$
\frac{1}{3}\|x\|^{2} \leq\|y\|^{2} \leq 3\|x\|^{2}
$$

Together with (A5), (A6) this implies (20), (21).

\section{REFERENCES}

[1] S. Arimoto, "Design of robot control systems," Advanced Robotics, vol. 4, pp. 79-91, 1990.

[2] H. Berghuis, "Model-based robot control: From theory to practice," $\mathrm{PhD}$ dissertation, Faculties of Electrical Engineering and Applied Mathematics, Univ. Twente, Enschede, Netherlands, 1993.

[3] H. Berghuis and H. Nijmeijer, "A passivity approach to controllerobserver design for robots," IEEE Trans. Robotics and Automation, vol. 9, pp. 940-954, 1993.

[4] C. Canudas de Wit, N. Fixot, and K. J. Åström, "Trajectory tracking in robot manipulators via nonlinear estimated state feedback," IEEE Trans. Robotics Automation, vol. 8, pp. 138-144, 1992.

[5] C. Canudas de Wit and N. Fixot, "Robot control via robust estimated state feedback," IEEE Trans. Automat. Contr., vol. 36, pp. 1497-1501, 1991.

[6] Y. H. Chen and G. Leitmann, "Robustness of uncertain systems in the absence of matching assumptions," Int. J. Contr., vol. 45, pp. 1527-1542, 1987

[7] J. J. Craig, Adaptive Control of Mechanical Manipulators. New York: Addison-Wesley, 1988.

[8] S. Kawamura, F. Miyazaki, and S. Arimoto, "Is a local linear PD feedback control law effective for trajectory tracking of robot motion?," in Proc. IEEE Conf. Robotics and Automation, 1988, pp. 1335-1340.

[9] R. D. Klafter, T. A. Chmielewski, and M. Negin, Robotic Engineering-An Integrated Approach. Englewood Cliffs, NJ: Prentice-Hall, 1989.

[10] D. E. Koditschek, "Strict global Lyapunov functions for mechanical systems," in Proc. Amer. Contr. Conf., 1988, pp. 1770-1775.

[11] S. Nicosia, and P. Tomei, "Robot control by using only joint position measurements," IEEE Trans. Automat. Contr., vol. 35, pp. 1058-1061, 1990.

[12] R. Ortega and M. W. Spong, "Adaptive motion control of rigid robots: A tutorial," Automatica, vol. 25, pp. 877-888, 1989.

[13] Z. Qu and J. F. Dorsey, "Robust tracking control of robots by a linear feedback law," IEEE Trans. Automat. Contr., vol. 36, pp. 1081-1084, 1991.

[14] M. W. Spong and M. Vidyasagar, Robot Dynamics and Control. New York: Wiley, 1989.

[15] X. Wang and L. K. Chen, "Proving the uniform boundedness of some commonly used control schemes for robots," in Proc. IEEE Conf. Robotics and Automation, 1989, pp. 1491-1496. 